\title{
Commentary: Control of thromboembolism and bleeding complications with mechanical heart valve prostheses (time in therapeutic range)
}

\author{
W. R. Eric Jamieson, MD, FRCS (C), FACS
}

\footnotetext{
From the Department of Surgery, University of British Columbia, and Vancouver Coastal Health, Research Institute, Vancouver, British Columbia, Canada.

Disclosures: Author has nothing to disclose with regard to commercial support.

Received for publication Aug 27, 2019; revisions received Aug 27, 2019; accepted for publication Aug 27, 2019. Address for reprints: W. R. Eric Jamieson, MD, FRCS (C), FACS, Department of Surgery, University of British Columbia, Vancouver Coastal Health, Research Institute, 2635 (6th floor) 2635 Laurel St, Vancouver, British Columbia V5Z 1M9, Canada (E-mail: eric.jamieson@vch.ca).

J Thorac Cardiovasc Surg 2020;159:86-7 $0022-5223 / \$ 36.00$

Copyright (C) 2019 Published by Elsevier Inc. on behalf of The American Association for Thoracic Surgery https://doi.org/10.1016/j.jtcvs.2019.08.106
}

The major complications of mechanical heart valve prostheses are thromboembolism and bleeding. In this issue of The Journal of Thoracic and Cardiovascular Surgery, Havers-Borgersen and colleagues have strived to evaluate a method of reduction of major complications related to the use of vitamin $\mathrm{K}$ antagonists. ${ }^{1}$ The major thromboembolic complications are prosthesis thrombosis, stroke, acute myocardial complication, and arterial embolism.

The authors have advanced an evaluation of anticoagulant therapeutic range during the initial 6 months after surgery to achieve reduction of thromboembolism and bleeding complications. The authors assessed international normalized ratio evaluations in 659 patients during the 6-month index period after surgery. The patients who experienced thromboembolism, hemorrhage, and death during completion of the index period were excluded in the analysis.

The time in therapeutic range (TTR) was determined in 2 groups: less than $70 \%$ and $70 \%$ or higher, determined according to international normalized ratio evaluations of 2-3 for aortic prostheses and 2.5-3.5 for mitral prostheses. The time in the therapeutic interval was determined with the total time of observation.

The lower-quality TTR of less than $70 \%$ revealed a higher level of thromboembolism compared with the higher-quality TTR of $70 \%$ or higher. The baseline characteristics were similar within the 2 groups of time in the therapeutic ranges. The TTR was lower for the mechanical mitral prostheses than the mechanical aortic prostheses. The incidence of thromboembolism and allcause mortality in the total mechanical heart valve population was greater in TTR of less than $70 \%$ compared with TTR of $70 \%$ or higher. The overall evaluation failed to reveal any difference in anticoagulant bleeding, defined by admission to hospitalization for bleeding complications.

Mechanical prostheses are of major importance in patients younger than 65 years of age. It is very considerate

\section{CONCLUSION}

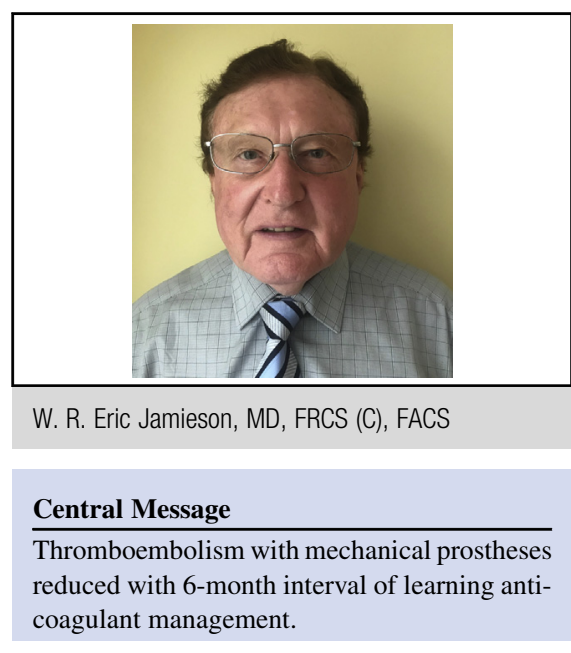

See Article page 74.

to place bioprostheses in patients of more advanced age whose life expectancy is more in keeping with advanced failure of bioprostheses. Valve-in-valve procedures are commonly performed for structural valve deterioration of aortic bioprostheses, more adaptable to failed aortic bioprostheses than mitral bioprostheses. The potential of valve-in-valve procedures for structural valve deterioration can only provide satisfactory hemodynamics with consideration of satisfactory size of the initial bioprostheses.

This study by Havers-Borgersen and colleagues is of extreme importance for the determination of control of thromboembolic complications of mechanical prostheses. The process of determining advanced therapeutic time range facilitates patients having a 6-month index period to learn the importance of proper anticoagulation management. The recommended anticoagulant levels used in this study are generally accepted levels for patient management of aortic and mitral mechanical prostheses.

The 6-month index time interval to learn the advancement of anticoagulant management is the major critical recommendation of this study. The recommendation is that all patients having the importance of the determination of advancement of therapeutic time intervals is extremely important in the early anticoagulant management with mechanical prostheses implantations. 


\section{Reference}

1. Havers-Borgersen E, Butt JH, Vinding NE, Torp-Pedersen C, Gislason G, Køber L, et al. Time in therapeutic range and risk of thromboembolism and bleeding in patients with a mechanical heart valve prosthesis. J Thorac Cardiovasc Surg. 2020;159:74-83.e4. 